\title{
Thermal desorption of methanol in hot cores. Study with a quartz crystal microbalance
}

\author{
Ramón Luna, ${ }^{\star}$ Miguel Ángel Satorre, ${ }^{\star}$ Manuel Domingo, Carlos Millán, ${ }^{\star}$ \\ Ramón Luna-Ferrándiz, Georgina Gisbert and Carmina Santonja \\ Centro de Tecnologías Físicas. Universitat Politècnica de València, Plaza Ferrándiz Carbonell, E-03801 Alcoy, Spain
}

Accepted 2017 September 22. Received 2017 September 21; in original form 2016 June 3

\begin{abstract}
The desorption process of methanol in the hot cores of massive young stars is addressed in this work. The study of pure methanol ice and when it is mixed or layered with water allows a better understanding of the physical and chemical processes which could have occurred during the formation of methanol and it is possible to infer the range of temperatures within which methanol can be found in the gas phase in these scenarios. The goal of this study was to model the desorption process of methanol as pure ice and mixed or layered with water under the conditions present in the early stages of hot cores whichcharacterize young star formation. The simulations of desorption of methanol, when it stands alone, performed in this work were compared to the values obtained by other authors to validate the method presented. In this work, the desorption of a water:methanol mixture under astrophysical conditions is also simulated. The theoretical results obtained for layered mixtures match with the temperatures at which an increase of the presence of methanol in the gas phase is detected when young massive mass stars are observed. This study has been performed using the frequency variation of a quartz crystal microbalance which provides a direct measure of the desorbing molecules during the experiments. This process was modelled using the Polanyi-Wigner equation and applied to astrophysical scenarios.
\end{abstract}

Key words: molecular data-molecular processes-methods: laboratory: solid state-ISM: molecules.

\section{INTRODUCTION}

Methanol $\left(\mathrm{CH}_{3} \mathrm{OH}\right)$ is reported to be one of the most abundant ices in interstellar matter. Its abundance has been detected from infrared spectral observations and extends from upper limits and weak detections (not more than a few percent in many lines of sight) to substantial levels of up to $\sim 30$ per cent relative to water ice towards young stellar objects (Sandford \& Allamandola 1993; Dartois et al. 1999; Pontoppidan et al. 2003; Gibb et al. 2004; Pontoppidan, van Dishoeck \& Dartois 2004; Whittet et al. 2011).

In space, the formation of three-atom molecules is not expected to directly take place in the gas phase (Whittet \& Chiar 1993); rather, they are thought to be assembled on solid surfaces such as dust grains. In the case of $\mathrm{CH}_{3} \mathrm{OH}$, this molecule is formed by the hydrogenation process which implies the presence of $\mathrm{CO}$ and $\mathrm{H}$ on grains (Charnley, Tielens \& Rodgers 1997; Tielens \& Whittet 1997; Watanabe \& Kouchi 2002; Fuchs et al. 2009). Another formation path for methanol has been reported by other authors through UVirradiation. For instance, Martín-Doménech, Muñoz-Caro \& Cruz-

\footnotetext{
^E-mail: ralunam@ fis.upv.es (RL); msatorre@ fis.upv.es (MAS); cmil-
} lan@ fis.upv.es (CM)
Diaz (2016) irradiated a $\mathrm{H}_{2} \mathrm{O}: \mathrm{CH}_{4}$ ice analogue with UV photons in the laboratory and obtained an amount of methanol representing an $\sim 4$ per cent of the initial $\mathrm{CH}_{4}$.

Once $\mathrm{CH}_{3} \mathrm{OH}$ ice is formed, it can be UV processed leading to more complex organic molecules (COMs). If any source of energy (a nearby star, dust grain collisions, etc.) is present, this molecule in the solid phase undergoes desorption and it can be detected in the gas phase as well. Photodesorption of methanol has been addressed by some authors but it seems to be negligible (Bertin et al. 2016; Cruz-Díaz et al. 2016). COMs such as methyl formate $\left(\mathrm{HCOOCH}_{3}\right)$ or dimethyl ether $\left(\mathrm{CH}_{3} \mathrm{OCH}_{3}\right)$ have been already detected in the Galactic Centre when grain mantles are sputtered by shocks (Requena-Torres et al. 2008). Additionally, $\mathrm{HCOOCH}_{3}$ and acetic acid $\left(\mathrm{HOCOCH}_{3}\right)$ were observed in lowmass hot cores (Bottinelli et al. 2004). Öberg et al. (2009) irradiated pure $\mathrm{CH}_{3} \mathrm{OH}$ in the laboratory and they obtained molecules like acetaldehyde $\left(\mathrm{CH}_{3} \mathrm{CHO}\right), \mathrm{CH}_{3} \mathrm{OCH}_{3}$, ethanol $\left(\mathrm{CH}_{3} \mathrm{CH}_{2} \mathrm{OH}\right)$, ethylene glycol $\left(\mathrm{CH}_{2} \mathrm{OH}\right)_{2}$ and mixtures of complex $\mathrm{CHO}$ - and $\mathrm{COOH}-$ containing molecules. Garrod \& Widicus Weaver (2013) simulated the hot-core chemistry, proposing several formation mechanisms for these species.

This study is focused on the thermal process of methanol in the early stages of hot cores which, at first, depend on the mass of the 
new forming star (Viti et al. 2004). Methanol has been observed towards low (Pontoppidan et al. 2003; Öberg et al. 2011a), intermediate (Kama et al. 2010) and massive (Dartois et al. 1999; van der Tak, van Dishoeck \& Caselli 2000) young stars, but the results show that the abundance of methanol observed does not depend on the mass of these objects, despite the fact that they have different temperatures and density.

Depending on the thermal process suffered by methanol, it will be available for a different period of time in the solid phase to form other species (COMs) during the hot-core stage.

The study of the desorption kinetics parameters of this molecule (Doronin et al. 2015) is relevant to better understand the evolution of the solid and gas phases present in the early stages of hot cores when the temperature starts to increase. The Polanyi-Wigner (Polanyi \& Wigner 1925) equation is widely used to model the desorption process of ices in space. When this model is used to describe a desorption process, it is necessary to have previously determined three different parameters: the desorption energy, the frequency factor and the desorption order. These values allow us to calculate the desorption rate and residence time, which both depend on the temperature.

Some authors have determined these parameters for the case of methanol using different techniques, mainly temperature programmed desorption (TPD) along with mass spectroscopy (Bolina, Wolff \& Brown 2005; Brown \& Bolina 2007; Smith, Matthiensen \& Kay 2014; Doronin et al. 2015) or infrared spectroscopy (Sandford \& Allamandola 1993). In these works, the authors assumed no variations of the frequency factor with the temperature in order to simplify the procedure.

The goal of this work is to obtain the desorption energy and the frequency factor in a more accurate manner in order to establish a model for the desorption rate of methanol when its evolution is governed by a zeroth-order process for a pure methanol ice and for methanol mixed with water ice, since it is commonly assumed that both molecules are mixed in the solid phase, with water being the main component.

Ices as solid $\mathrm{H}_{2} \mathrm{O}$ and $\mathrm{CO}$ are formed sequentially in dark clouds and protostars, resulting in a bi-layered structure dominated by water and carbon monoxide, respectively ( $\mathrm{CO}$ being dominant in the external layer). As methanol is formed in the pre-stellar phase, it is probably present in a CO-rich phase during most of its lifetime (Öberg et al. 2009, 2011a), therefore methanol and water would be present in different layers. As some ices can be liberated by shocks from the dust grains (Requena-Torres et al. 2008), this would lead to a further co-deposition and the formation of a new structure where water and methanol could be mixed. Consequently, the desorption temperature of methanol could differ depending on how this molecule is mixed with water.

This article is presented as follows: in Section 2, the experimental set-up is described. In Section 3, the results for the desorption of pure methanol are presented, and Section 4 contains the results on the desorption of methanol mixed with water ice. In Section 5, the astrophysical implications are discussed and in Section 6, the conclusions are presented.

\section{EXPERIMENTAL SET-UP}

All experiments performed in this study were carried out in a high-vacuum (HV), low-temperature system, on the basis of which several analysis techniques were implemented: a quartz crystal microbalance (QCMB), an interferometric system (IS) and a quadrupole mass spectrometer (QMS).
The vacuum in the chamber is obtained via two turbo-molecular pumps (working in parallel), each backed by a rotary pump. The first stage of a closed-cycle He cryostat $(40 \mathrm{~K})$ acts as a cryopump to provide a base pressure below $10^{-7} \mathrm{mbar}$, as measured with an ITR IoniVac transmitter ( 5 per cent in accuracy, Pfeiffer vacuum).

The composition of the gas present in the chamber is monitored with the QMS (AccuQuad RGA 100 with a resolution of $\sim 0.5 \mathrm{amu}$ and an ionization energy of $70 \mathrm{eV}$ ) throughout the experiment.

The QCMB is located below the second stage in the sample holder. This configuration reaches $13 \mathrm{~K}$ at the sample holder, in thermal contact with the QCMB (Q-Sense X 301; AT-cut; 0216/1; $5 \mathrm{MHz}$ ). In this experimental set-up, it is worth noting that molecules sublimate directly from this QCMB, which acts as both a sample holder and a sensor.

The temperature of the sample (QCMB) is controlled by the Intelligent Temperature Controller ITC 503S (Oxford Instruments) using feedback from a silicon diode sensor (Scientific Instruments) located just beside the quartz, which allows the temperature to be varied by means of a resistive heater with an accuracy of $0.5 \mathrm{~K}$.

The gas or vapour of the molecule under study is charged in a prechamber at a suitable pressure, as measured with a Ceravac CTR 90 (Leybold vacuum) with an accuracy of 0.2 per cent, provided with a ceramic sensor not influenced by the gas type (range $10^{4}, \mathrm{FS}$ 100 torr). Gas enters the chamber through a needle valve (Leybold D50968) which regulates its flow. During deposition, the pressure of the molecule studied is at least three orders of magnitude greater than the residual pressure in the chamber, which ensures a negligible quantity of contaminants in the ice film and leads to a decrease in frequency of approximately $10 \mathrm{~Hz} \mathrm{~s}^{-1}$. Gas fills the chamber and is deposited on to the QCMB from random directions, this is known as background deposition. The interferometric patterns of an $\mathrm{He}-\mathrm{Ne}$ $(632.8 \mathrm{~nm})$ IS are used, aimed at the same surface, to determine the thickness of the ice deposited. Once a film depth of around $2.0 \mu \mathrm{m}$ is reached, the ice is desorbed at a constant rate of $2 \mathrm{~K} \mathrm{~min}^{-1}$, which is controlled by the ITC.

The methanol used in this study was 99.9 percent (Panreac), and ultrapure water was obtained from an Elix 3 Millipore-MilliQ Advantage A10 system with a resistivity of nearly $18.2 \mathrm{M} \Omega \mathrm{cm}$. Both compounds are distilled before deposition to remove dissolved gases. To preserve gas purity, the pre-chamber is evacuated with a turbo-drag pumping station (ultimate pressure $<10^{-6}$ mbar).

\section{DESORPTION OF PURE METHANOL}

Desorption of pure ices is widely modelled using the PolanyiWigner equation

$r_{\mathrm{des}}=-\frac{\mathrm{d} N}{\mathrm{~d} t}=A_{i} N^{i} \exp \left(-\frac{E_{\mathrm{des}}}{R T}\right)$

where $r_{\text {des }}$ is the desorption rate (number of molecules per square centimetre which leave the frozen surface), $i$ is the order of the process, $A_{i}$ is the frequency factor for the corresponding order $i, N$ is the number of molecules per square centimetre on the surface, $R$ is the constant of ideal gases, $E_{\mathrm{des}}$ is the desorption energy and $T$ is the absolute temperature of the surface.

For multilayer desorption processes, as was the case under the experimental conditions of this work, a zeroth-order desorption process is involved (i.e. when the top surface desorbs, another monolayer with the same characteristics appears), therefore, the previous 


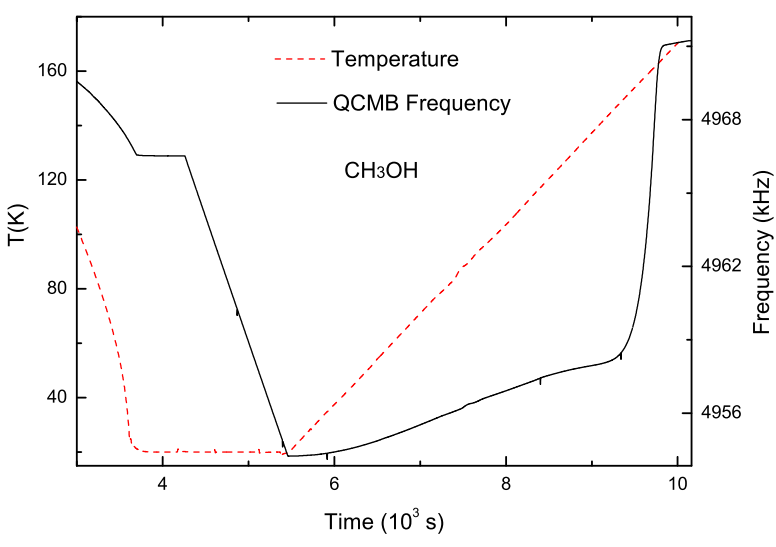

Figure 1. Solid line: raw signal of the QCMB throughout the whole experiment for $\mathrm{CH}_{3} \mathrm{OH}$. Dashed line: profile of the temperature throughout the experiment.

equation becomes

$r_{\mathrm{des}}=-\frac{\mathrm{d} N}{\mathrm{~d} t}=A_{0} \exp \left(-\frac{E_{\mathrm{des}}}{R T}\right)$

To use this equation, the desorption energy $\left(E_{\mathrm{des}}\right)$ and the frequency factor $\left(A_{0}\right)$ must be determined. So far, other authors have obtained both parameters from the same experiment assuming that $A_{0}$ is not influenced by the temperature. Instead, to determine the value of this parameter and its temperature dependence, a procedure based on two different types of experiments has been developed and described in Sections 3.1 and 3.2.

\subsection{Desorption energy}

Desorption energy was determined performing desorption at a constant warming rate after methanol deposition. In Fig. 1, the raw signals recorded during deposition and warming for $\mathrm{CH}_{3} \mathrm{OH}$ are shown. Here, the dashed line represents temperature and the solid line shows QCMB frequency.

There are two characteristic zones in the graph. First, in the interval from 4500 to $5500 \mathrm{~s}$, the deposition is performed. At the starting point of deposition $(\sim 4500 \mathrm{~s})$, the frequency signal starts to fall (solid line in Fig. 1) with a constant slope due to a constant mass deposition rate on to the QCMB. When a film thickness of approximately $2.0 \mu \mathrm{m}$ is achieved (determined by means of the interference fringes obtained by the IS), the deposition is stopped. Secondly, the warming-up process starts at approximately $5500 \mathrm{~s}$ in Fig. 1, when the dashed line (temperature) and the solid line (QCMB frequency) start to rise. Warming starts when the deposition ends to prevent contaminants. A constant heating rate of $2 \mathrm{~K} \mathrm{~min}^{-1}$ was used. The solid line in Fig. 1, within the interval 5500-9500 s, represents how the frequency of the QCMB changes due to three simultaneous events: desorption of molecules from the QCMB, frequency dependence on temperature and deposition of contaminants (mainly water). After removing the two last contributions, from the signal recorded in this interval, and calculating the derivative with respect to the time, the desorption rate versus temperature (Fig. 2, solid line) is obtained, which is governed by the Polanyi-Wigner equation (equation 2). The overall procedure is more detailed in Luna et al. (2009).

From the film thickness, it is possible to roughly estimate that the film is formed by few thousands monolayers of molecules. Therefore, the desorption must be governed by a zeroth-order process where no substrate effects are relevant, as is generally assumed for multilayer films (Smith et al. 2014). In Fig. 2, a comparison between

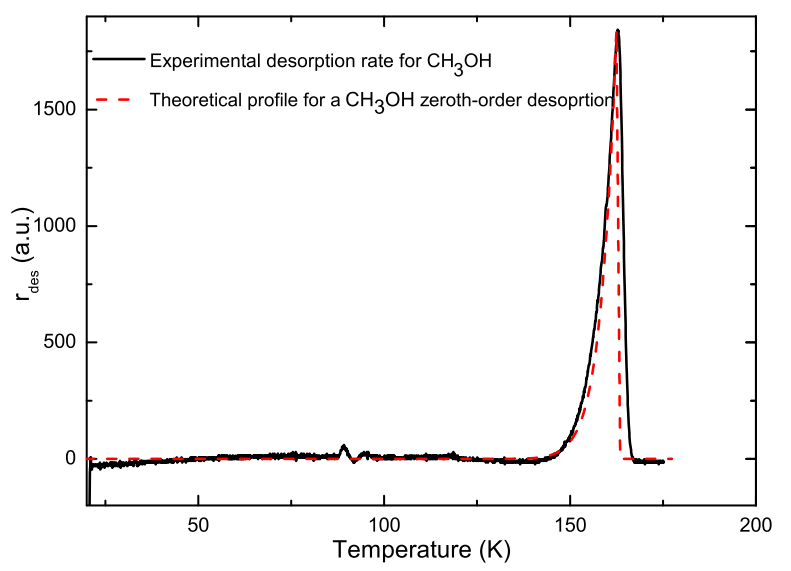

Figure 2. Solid line: experimental desorption rate of methanol. Dashed line: theoretical profile of methanol following a zeroth-order desorption.

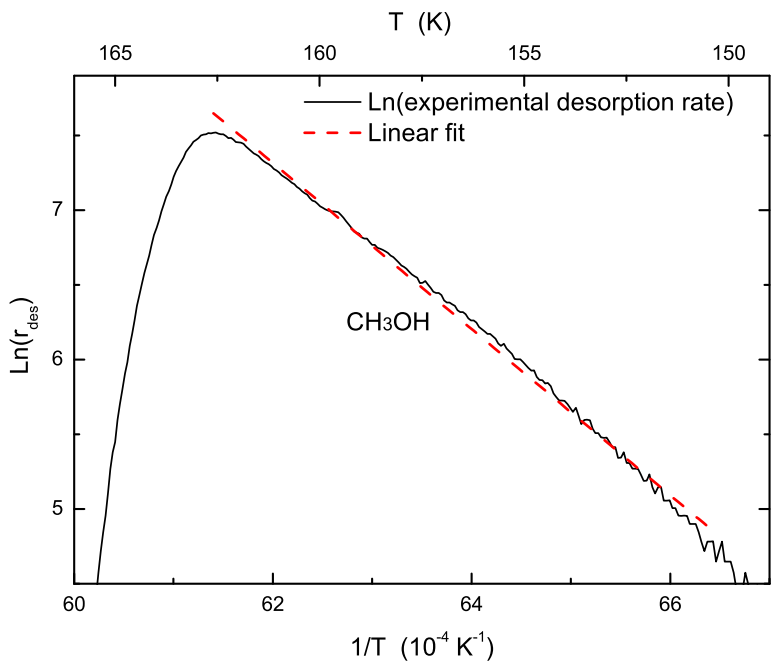

Figure 3. Solid line: natural logarithm of the desorption rate. Dashed line: fit to a straight line to obtain $E_{\text {des }}$.

experimental desorption rate for methanol (solid line) and theoretical desorption assuming a zeroth-order process (dashed line) is shown. The strong similarities between both profiles confirm the zeroth-order desorption process for methanol under the experimental conditions of this work. Under these circumstances, $\mathrm{CH}_{3} \mathrm{OH}$ desorption peaks at $162 \mathrm{~K}$.

In a further step, taking the natural logarithm for the PolanyiWigner equation for a zeroth-order desorption,

$\ln \left(r_{\text {des }}\right)=\ln \left(A_{0}\right)-\frac{E_{\text {des }}}{R} \frac{1}{T}$,

the desorption energy from the slope of the fit obtained with a constant rate of warming up is calculated (Fig. 3, dashed line). For this calculation, as a first approach, it was assumed that the $A_{0}$ temperature dependence does not influence the value of the desorption energy. For methanol, the value obtained for the desorption energy was $E_{\mathrm{des}}=46.2 \pm 2.4 \mathrm{~kJ} \mathrm{~mol}^{-1}$. The uncertainty was calculated from five different experiments.

In Table 1, the results of this work are compared to others reported in the literature using different methods and techniques, for zerothorder desorption processes. As can be observed, the results presented here are comparable to those of other researchers. The main discrepancy can be observed in the case of Sandford \& Allamandola 
Table 1. Desorption energy and peak reported by different authors for multilayer desorption.

\begin{tabular}{lcc}
\hline Reference & $\begin{array}{c}\text { Desorption } \\
\text { energy } \\
\left(\mathrm{kJ} \mathrm{mol}^{-1}\right)\end{array}$ & $\begin{array}{c}\text { Desorption } \\
\text { peak } \\
(\mathrm{K})\end{array}$ \\
\hline This work & $46.2 \pm 2.4$ & 162 \\
Doronin et al. (2015) & $41.5 \pm 2.9$ & 146 \\
Smith et al. (2014) & $45.4 \pm 2.3$ & 146 \\
Brown and Bolina (2007) & $40.99 \pm 0.81$ & 159 \\
Bolina et al. (2005) & $48.2 \pm 0.35$ & 154 \\
Sandford and Allamandola (1993) & $35.20 \pm 0.12$ & $150^{a}$ \\
\hline$a_{\text {Their highest temperature used for calculations. }}$ &
\end{tabular}

${ }^{a}$ Their highest temperature used for calculations.

(1993). Some authors (Bolina et al. 2005; Brown \& Bolina 2007; Smith et al. 2014; Doronin et al. 2015) used mass spectroscopy during TPD experiments with a base pressure of $\sim 1 \times 10^{-10} \mathrm{mbar}$ (UHV conditions). Their samples were grown at temperatures in the interval $25-100 \mathrm{~K}$ (depending on the author), and then warmed up using rates ranging from 1 to $6 \mathrm{~K} \mathrm{~min}^{-1}$. Their desorption experiments corresponding to multilayer films peaked in the range 146-159 K. On the other hand, Sandford \& Allamandola (1993) used infrared spectroscopy in a HV system whose base pressure is $\sim 3 \times 10^{-8}$ mbar. The ice film was grown at $10 \mathrm{~K}$ and then was warmed up at $2 \mathrm{~K} \mathrm{~min}^{-1}$ until the desired desorption temperature was reached and maintained constant during desorption. They carried out two desorption experiments at two different temperatures: 140 and $144 \mathrm{~K}$.

Concerning the temperature for the desorption peak, with the method presented in this work the highest value is obtained. This result is the one expected in a typical zeroth-order desorption since the peak depends on the thickness of the film. Therefore, because the coverage in this experiment is larger than for the other authors (due to pressure conditions and time of deposition), the peak is expected to appear at higher temperatures. Sandford \& Allamandola (1993) did not report the peak temperature. Nevertheless, from their work it is possible to infer that $150 \mathrm{~K}$ is the highest temperature they were able to work under stable experimental conditions.

\subsection{Frequency factor $\left(A_{0}\right)$}

To obtain $A_{0}$, additional sets of experiments were carried out in this work. They consisted of a series of desorption experiments performed at several selected constant temperatures close to those of desorption. This procedure also allows the influence of the temperature on this parameter (Galwey \& Brown 2002; Luna et al. 2015) to be studied. First, the ice is grown at low temperature, then, the sample is warmed at $2 \mathrm{~K} \mathrm{~min}^{-1}$ until a temperature close to the desorption temperature is reached, and is maintained constant during the rest of the experiment. The closer this temperature is to that of desorption, the faster the film desorbs. Performing a thick deposit ensures an amount of experimental points large enough (longer than $300 \mathrm{~s}$ ) to reduce the uncertainty as much as possible.

According to equation (2), if the temperature remains invariable during the experiment, a constant desorption rate occurs, and then plotting QCMB frequency signal versus time must correspond to a straight line with a positive slope. The experimental points obtained in this work are plotted in Fig. 4 as a solid line. The correlation factor of the corresponding linear fit (dashed line) is higher than 0.999 for the experimental desorption of methanol at $152 \mathrm{~K}$. This is an additional argument to demonstrate that under the experimental conditions of this work, desorption of methanol follows a zeroth-

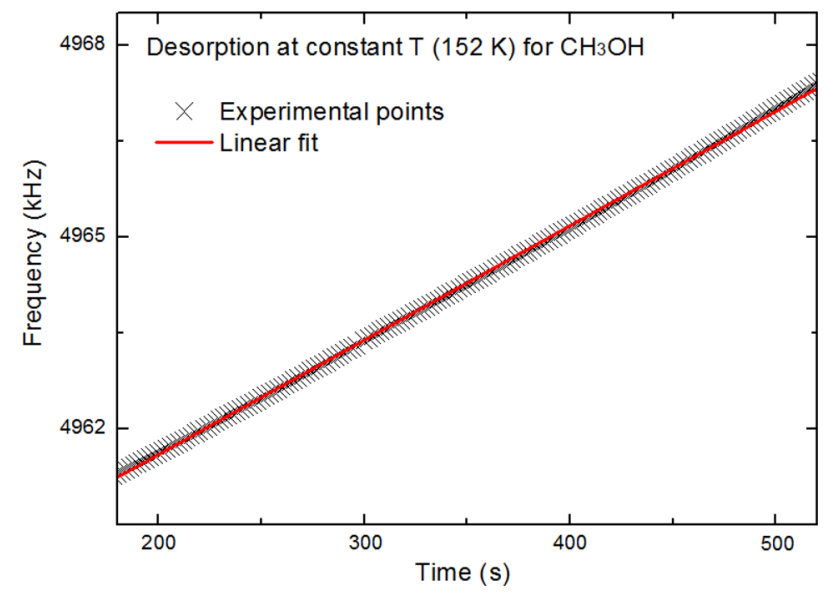

Figure 4. QCMB signal obtained during desorption at $152 \mathrm{~K}$ (solid line). Linear fit (dashed line) with a correlation parameter $r>0.999$.

Table 2. Different values of $A_{0}$ for methanol, obtained for each temperature using the Polanyi-Wigner equation.

\begin{tabular}{lc}
\hline $\begin{array}{l}\text { Temp. } \\
(\mathrm{K})\end{array}$ & $\begin{array}{c}A_{0} \\
\left(10^{31} \text { molecule } \mathrm{cm}^{-2} \mathrm{~s}^{-1}\right)\end{array}$ \\
\hline 144 & $1.29 \pm 0.09$ \\
146 & $1.47 \pm 0.10$ \\
151 & $1.81 \pm 0.13$ \\
152 & $2.17 \pm 0.15$ \\
\hline
\end{tabular}

order process. The set of temperatures used to perform the $\mathrm{CH}_{3} \mathrm{OH}$ desorption experiments were 144, 146, 151 and $152 \mathrm{~K}$.

From the slope of the QCMB signal obtained during these experiments, and assuming no significant variation for $E_{\mathrm{des}}$ with temperature (since different authors at different temperatures obtain similar results), $A_{0}$ is derived for each temperature from equation (4):

$A_{0}=r_{\mathrm{des}} \exp \left(\frac{E_{\mathrm{des}}}{R T}\right)$

where $r_{\text {des }}$ were measured experimentally for each experiment at the temperatures referred to above.

Table 2 shows the experimental values obtained with this analysis. Column 1 represents the temperature at which the experiments were performed and in column 2 the corresponding value of $A_{0}$ is presented. These values are also plotted in Fig. 5, where a temperature dependence is observed. An expression relating the dependence of $A_{0}$ with the temperature was obtained from the linear fit (solid line in Fig. 5):

$A_{0}=0.97 T-126\left(10^{+30}\right.$ molecule $\left.\mathrm{cm}^{-2} \mathrm{~s}^{-1}\right)$

This expression is valid at least for the interval of temperatures from 144 to $152 \mathrm{~K}$, in which a measurable desorption rate occurs in our experiment. However, $A_{0}$ cannot be experimentally determined for all the temperatures due to the experimental limitations. Nevertheless, using the expression obtained, and assuming that it can be extrapolated at least to the range of temperatures 140-165 K (with $162 \mathrm{~K}$ the desorption temperature for $\mathrm{CH}_{3} \mathrm{OH}$ under the experimental conditions of this work), it is possible to calculate the residence time for methanol for temperatures within this interval to analyse the influence on $A_{0}$. Lower temperatures than $140 \mathrm{~K}$ are not used in this study since desorption rate dramatically decreases below this temperature under our experimental conditions and it can be 


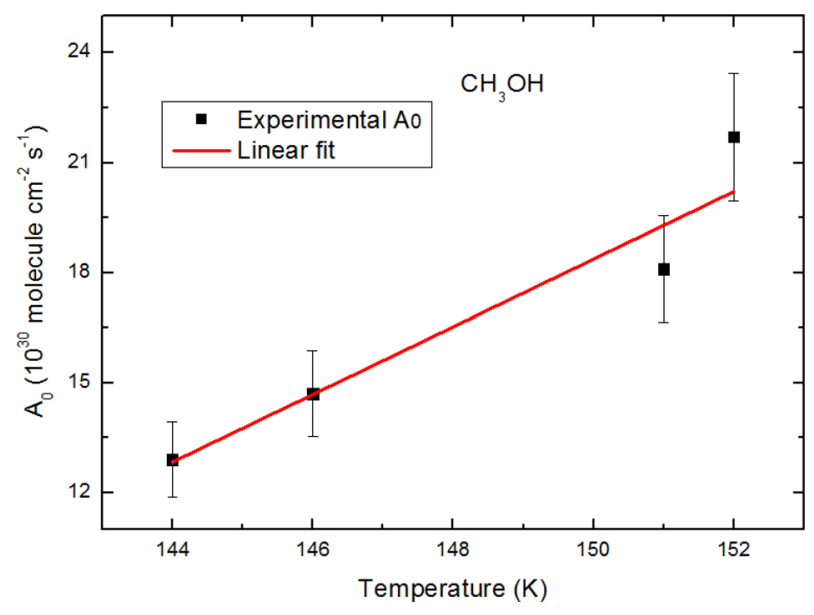

Figure 5. Experimental $A_{0}$ values obtained at different constant temperatures (filled squares). Linear fit (solid line).

Table 3. Half-life calculated at different temperatures, assuming $A_{0}$ constant (column 2) or using $A_{0}$ as a function of the temperature (column 3 ). For both cases, a number of molecules of $1.15 \times 10^{17}$ molecule $\mathrm{cm}^{-2}$ was used.

\begin{tabular}{lcc}
\hline & \multicolumn{2}{c}{ Half-life $(\mathrm{yr})$} \\
Temperature $(\mathrm{K})$ & $A_{0}=$ ct. & $A_{0}=f(T)$ \\
\hline 10 & $1.20 \times 10^{+220}$ & $1.20 \times 10^{+220}$ \\
50 & $8.32 \times 10^{+26}$ & $8.32 \times 10^{+26}$ \\
90 & $2.87 \times 10^{+05}$ & $2.87 \times 10^{+05}$ \\
130 & $1.60 \times 10^{-03}$ & $1.60 \times 10^{-03}$ \\
140 & $7.53 \times 10^{-05}$ & $3.53 \times 10^{-05}$ \\
150 & $5.33 \times 10^{-06}$ & $1.21 \times 10^{-06}$ \\
160 & $52.6 \times 10^{-08}$ & $7.91 \times 10^{-08}$ \\
165 & $18.4 \times 10^{-08}$ & $2.36 \times 10^{-08}$ \\
\hline
\end{tabular}

considered as negligible as can be seen in Fig. 2. Nevertheless, under astrophysical conditions, this desorption temperature decreases down to $90 \mathrm{~K}$ as can be seen in Section 4 from our simulation and observational results due to a significantly lower warming rate (from $0.5 \mathrm{~K} \mathrm{~min}^{-1}$ in the laboratory up to $1 \mathrm{~K}$ century $^{-1}$ in some astrophysical scenarios).

The parameter half-life is widely used as representative of residence time of a species in a certain astrophysical scenario. For a zeroth-order desorption process, this parameter is calculated for each temperature as follows:

$t_{1 / 2}=\frac{0.5 N}{r_{\mathrm{des}}}$

where $N$ is the initial amount of molecules per square centimetre. For comparison purposes only, a value of $1.15 \times 10^{17}$ molecule $\mathrm{cm}^{-2}$ was taken as the initial number of molecules of pure ice of methanol because this was the value used by Fraser et al. (2001) for water molecules. This amount is commonly used and represents approximately 100 monolayers of ice, which is the number of molecules expected to accrete on dust grains during the lifetime of a dark cloud (Hasegawa \& Herbst 1993).

Table 3 is intended to compare the results obtained for half-life of methanol ice in frozen grains of the ISM assuming a constant frequency factor (column 2) or using a frequency factor depending on temperature (column 3) and using the value of desorption energy experimentally determined in this work. The value used for $A_{0}$ in column 2 for all the temperatures is the correspond-

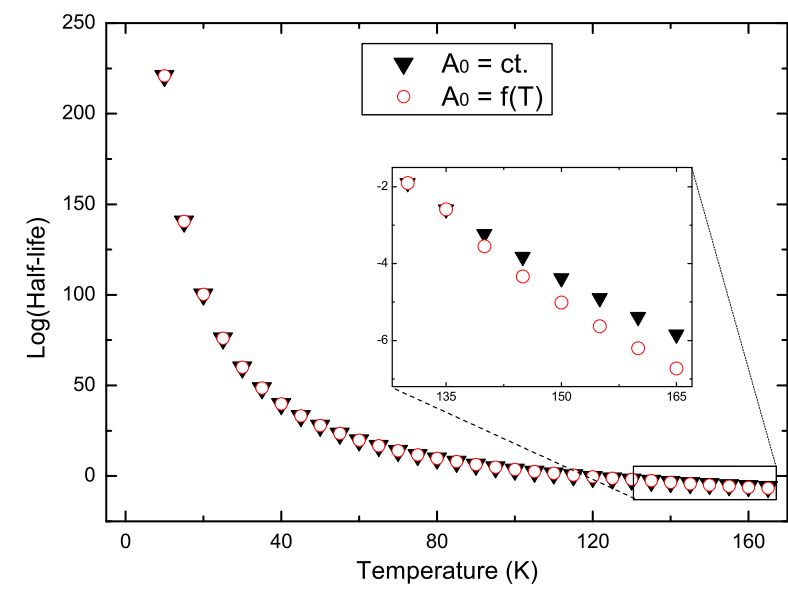

Figure 6. Comparison of half-life for methanol versus temperature, calculated assuming a constant (inverted filled triangles) or temperaturedependent factor frequency (open circles).

Table 4. Pre-exponential factor reported by different authors.

\begin{tabular}{lc}
\hline Reference & $\begin{array}{c}\text { Pre-exponential factor } \\
\left(\text { molecule } \mathrm{cm}^{-2} \mathrm{~s}^{-1}\right)\end{array}$ \\
\hline This work $(T \geq 140 \mathrm{~K})$ & $9.7 \times 10^{+29} \mathrm{~T}-1.26 \times 10^{+32}$ \\
Doronin et al. (2015) & $5.75 \times 10^{+31 a, b}$ \\
Smith et al. (2014) & $3.57 \times 10^{+33 a, c}$ \\
Brown and Bolina (2007) & $6 \times 10^{+25 \pm 3 c}$ \\
Bolina et al. (2005) & - \\
Sandford and Allamandola (1993) & $2.53 \times 10^{+29 a, d}$ \\
\hline
\end{tabular}

${ }^{a}$ The value reported in their work corresponds to a first-order desorption. $A_{0}=A_{1} \cdot N$ has been used.

${ }^{b}$ Obtained by means a linear fit of the linearized Polanyi-Wigner equation.

${ }^{c}$ Obtained by means a direct fit of the desorption curve.

${ }^{d}$ Determined from the infrared spectra of methanol in a matrix.

ing value obtained from equation (4) for a temperature of $140 \mathrm{~K}$ $\left(A_{0}=4.27 \times 10^{30}\right.$ molecule $\left.\mathrm{cm}^{-2} \mathrm{~s}^{-1}\right)$. The values used for $A_{0}$ in column 3 are $A_{0}=4.27 \times 10^{30}$ molecule $\mathrm{cm}^{-2} \mathrm{~s}^{-1}$ for temperatures lower than $140 \mathrm{~K}$, but for temperatures equal to or higher than $140 \mathrm{~K}$ the corresponding value obtained from equation (5) was used. These values are also plotted in Fig. 6 in a semilogarithmic scale. As can be seen in Table 3, as temperature increases, the half-life for both columns reaches a difference close to one order of magnitude at $165 \mathrm{~K}$. Therefore to obtain more accurate results from the desorption process for pure methanol, the influence of temperature in the pre-exponential factor has to be taken into account.

Concerning the values obtained from other authors for this parameter, Brown \& Bolina (2007), Smith et al. (2014) and Doronin et al. (2015) obtained $A_{0}$ (assumed as a constant) from the same experiment they performed to obtain $E_{\mathrm{des}}$. Sandford \& Allamandola (1993) obtained the pre-exponential factor from the infrared spectra during an additional experiment of methanol in matrices of hydroquinone and argon at a constant temperature. These values have been compiled in Table 4. For clarity, when the value reported by some authors corresponds to a first-order process, this parameter has been transformed to be related to a zeroth-order desorption using $A_{0}=A_{1} \cdot N$, where $N$ is the number of molecules per square centimetre, in this case $N=1.15 \times 10^{17}$ molecule $\mathrm{cm}^{-2}$, for the reason mentioned previously. 
Table 5. Calculated half-life for methanol at different temperatures for a coverage of $1.15 \times 10^{17}$ molecule cm ${ }^{-2}$.

\begin{tabular}{lccccc}
\hline Temperature (K) & This work & Doronin et al. (2015) & $\begin{array}{c}\text { Half-life (yr) } \\
\text { Smith et al. (2014) }\end{array}$ & $\begin{array}{c}\text { Brown and } \\
\text { Bolina (2007) }\end{array}$ & $\begin{array}{c}\text { Sandford and } \\
\text { Allamandola (1993) }\end{array}$ \\
\hline 10 & $1.20 \times 10^{+220}$ & $2.16 \times 10^{+194}$ & $5.19 \times 10^{+212}$ & $5.05 \times 10^{+197}$ & $6.59 \times 10^{+163}$ \\
20 & $2.26 \times 10^{+99}$ & $8.28 \times 10^{+85}$ & $1.63 \times 10^{+94}$ & $3.92 \times 10^{+90}$ & $6.89 \times 10^{+71}$ \\
30 & $1.30 \times 10^{+59}$ & $6.01 \times 10^{+49}$ & $5.13 \times 10^{+54}$ & $7.75 \times 10^{+54}$ & $1.51 \times 10^{+41}$ \\
40 & $9.83 \times 10^{+38}$ & $5.12 \times 10^{+31}$ & $9.12 \times 10^{+34}$ & $1.09 \times 10^{+37}$ & $7.05 \times 10^{+25}$ \\
50 & $8.32 \times 10^{+26}$ & $7.38 \times 10^{+20}$ & $1.29 \times 10^{+23}$ & $2.12 \times 10^{+26}$ & $4.47 \times 10^{+16}$ \\
60 & $7.45 \times 10^{+18}$ & $4.37 \times 10^{+13}$ & $1.62 \times 10^{+15}$ & $1.54 \times 10^{+19}$ & $3.30 \times 10^{+10}$ \\
70 & $1.33 \times 10^{+13}$ & $3.00 \times 10^{+08}$ & $3.68 \times 10^{+09}$ & $1.22 \times 10^{+14}$ & $1.37 \times 10^{+06}$ \\
80 & $6.48 \times 10^{+08}$ & $4.03 \times 10^{+04}$ & $2.16 \times 10^{+05}$ & $1.82 \times 10^{+10}$ & $7.13 \times 10^{+02}$ \\
90 & $2.87 \times 10^{+05}$ & $3.92 \times 10^{+01}$ & $1.10 \times 10^{+02}$ & $1.93 \times 10^{+07}$ & $1.99 \times 10^{+00}$ \\
100 & $5.96 \times 10^{+02}$ & $1.53 \times 10^{-01}$ & $2.56 \times 10^{-01}$ & $8.03 \times 10^{+04}$ & $1.79 \times 10^{-02}$ \\
110 & $3.80 \times 10^{+00}$ & $1.63 \times 10^{-03}$ & $1.80 \times 10^{-03}$ & $9.06 \times 10^{+02}$ & $3.81 \times 10^{-04}$ \\
120 & $5.64 \times 10^{-02}$ & $3.72 \times 10^{-05}$ & $2.88 \times 10^{-05}$ & $2.16 \times 10^{+01}$ & $1.54 \times 10^{-05}$ \\
130 & $1.60 \times 10^{-03}$ & $1.52 \times 10^{-06}$ & $8.70 \times 10^{-07}$ & $9.15 \times 10^{-01}$ & $1.02 \times 10^{-06}$ \\
140 & $3.53 \times 10^{-05}$ & $9.76 \times 10^{-08}$ & $4.34 \times 10^{-08}$ & $6.08 \times 10^{-02}$ & $9.95 \times 10^{-08}$ \\
150 & $1.21 \times 10^{-06}$ & $9.05 \times 10^{-09}$ & $3.23 \times 10^{-09}$ & $5.81 \times 10^{-03}$ & $1.32 \times 10^{-08}$ \\
160 & $7.91 \times 10^{-08}$ & $1.13 \times 10^{-09}$ & $3.32 \times 10^{-10}$ & $7.44 \times 10^{-04}$ & $2.27 \times 10^{-09}$ \\
165 & $2.36 \times 10^{-08}$ & $4.39 \times 10^{-10}$ & $1.18 \times 10^{-10}$ & $2.92 \times 10^{-04}$ & $1.02 \times 10^{-09}$ \\
\hline
\end{tabular}

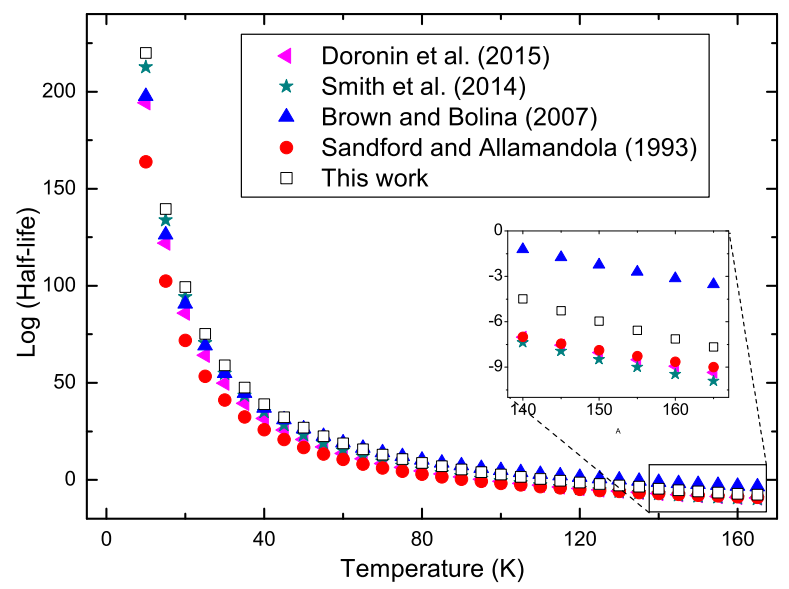

Figure 7. Half-life for methanol obtained at different temperatures by different authors.

\subsection{Desorption rate $\left(r_{\mathrm{des}}\right)$}

When the solid-gas phase kinetics of methanol is addressed, desorption energy and pre-exponential factor have to be studied together, as both are necessary in the Polanyi-Wigner equation. In order to compare the kinetics parameters obtained in this work with those reported by other authors, the half-life for methanol ice at different temperatures was calculated. In Table 5, this parameter for the different works previously cited is shown except for that of Bolina et al. (2005), as they have not reported this value. To enable comparison, these values have also been plotted in Fig. 7 in a semilogarithmic scale. To the first order, these results appear similar, as could be expected due to their similar values of $E_{\mathrm{des}}$. Smith et al. (2014) state that this result is what could be expected for a compensation effect between the two parameters involved $\left(E_{\mathrm{des}}\right.$ and $\left.A_{0}\right)$ in the PolanyiWigner equation. Nevertheless, in the zoom panel of Fig. 7, it is clear how the results obtained in this work tend to decrease faster than the rest at high temperatures, which confirms that for these temperatures more accurate results are obtained taking into account the temperature influence on the pre-exponential factor. These results on methanol, in agreement with other studies, validate the method

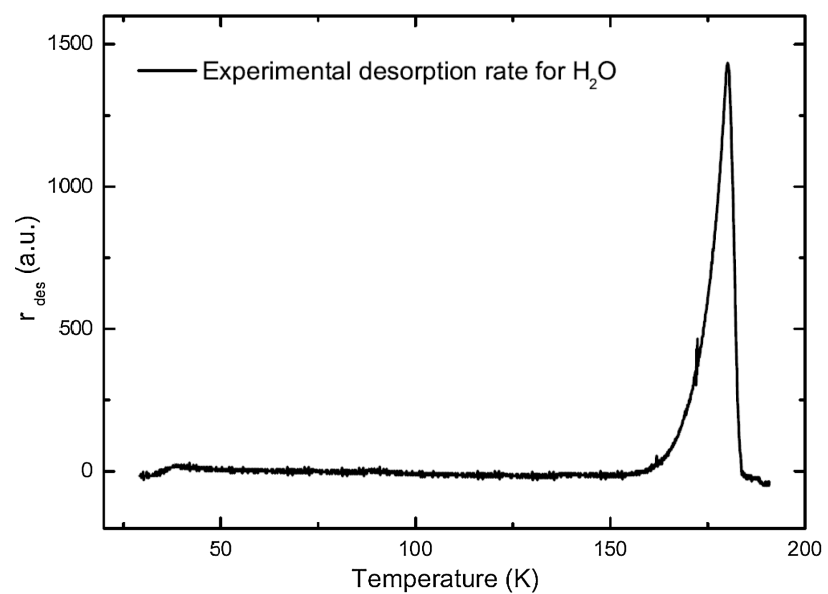

Figure 8. Experimental desorption rate of water.

and procedure presented here to calculate the desorption rate for ice species under astrophysical conditions and can be applied to the mixture methanol:water.

\section{DESORPTION OF THE MIXTURE METHANOL: WATER}

Once the desorption process of pure methanol ice is studied and this method is validated, it is possible to focus on a more complex system. Methanol ice has been detected in the hot cores of young massive stars. However, water is the most abundant ice species, therefore, desorption of methanol ice covering dust grains of hot cores must be affected by the major component and it is worthwhile to study how this mixture would evolve in this astrophysical scenario.

To undertake this study, it is necessary to determine separately the desorption kinetics for each pure ice. The kinetics parameters for water ice have been obtained following the same procedure used previously for methanol, from the desorption experiment (Fig. 8) of the former molecule. For this molecule, the following values were experimentally obtained, $E_{\mathrm{des}}=47.0 \pm 2.4 \mathrm{~kJ} \mathrm{~mol}^{-1}$, and 
$A_{0}=9.1 \times 10^{29}$ molecule $\mathrm{cm}^{-2} \mathrm{~s}^{-1}$. Within the range of temperatures of interest in this study, the desorption process of this molecule is negligible.

The desorption behaviour of a mixture of water and methanol was experimentally addressed by Cruz-Diaz, Martín-Doménech \& Muñoz (2004) using a mixture (100:5) and it was also studied by Martín-Doménech et al. (2014) using a similar mixture (100:3). Collings et al. (2004) performed two different kinds of desorption experiments. In the first experiment, methanol is deposited on to a pre-adsorbed water film (layered deposition). In this case, desorption of methanol is reported to occur at two different temperatures (Fig. 2 in Collings et al. 2004). The first one is the corresponding temperature at which methanol desorbs when it is deposited alone and it is assigned to a desorption corresponding to the methanolmethanol binding energy. The second desorption feature appears at a slightly higher temperature corresponding to a desorption at which the influence of the methanol-water binding energy is manifested. In the second experiment (fig. 3 in Collings et al. 2004), methanol and water are co-deposited; in this case, methanol only desorbs during the desorption of water ice, and it is interpreted as the molecules of methanol are trapped in the water matrix. MartínDoménech et al. (2014) obtained a simultaneous desorption for the co-deposition experiment for the mixture water:methanol (100:3) as occurred in the case of Collings et al. (2004).

Methanol is expected to be formed in the outer layers of the icy mantles (Öberg et al. 2009, 2011a); therefore the first experiment of Collings et al. (2004) could be seen as the most representative of the desorption process of this mixture, but the occasional occurrence of some energy inputs could lead to the abrupt desorption of the ices and further co-deposition of all of the previously desorbed molecules. Both scenarios (stratification versus mixed ices) must then be taken into consideration to interpret the presence of methanol in the solid and gas phases.

To study this mixture, we simulated the desorption of methanol presented by Collings et al. (2004) (water:methanol, 100:5) under the warming up conditions present in hot cores $1 \mathrm{~K}$ century ${ }^{-1}$, as assumed by Viti et al. (2004). The warming rate strongly influences the desorption temperature. This fact can be observed in Fig. 11 where a simulation of the desorption of water at several values of warming up $(\beta=\mathrm{d} T / \mathrm{d} t)$ was performed. This figure can show how as $\beta$ decreases, the temperature at which all the mantle desorb decreases.

Using a constant warming up $\beta=\mathrm{d} T / \mathrm{d} t$ in equation (1), it is obtained:

$$
-\frac{\mathrm{d} N}{\mathrm{~d} T}=N^{i} \frac{A_{i}}{\beta} \exp \left(-\frac{E_{\mathrm{des}}}{R T}\right)
$$

The first layers of methanol adsorbed on water are experimentally observed to desorb after a first-order process (Fig. 2 in Collings et al. 2004); therefore, both zeroth- and first-order desorption must be addressed. The number of molecules in the solid phase from equation (7) can be obtained. For a zeroth-order desorption process, the following expression has been used:

$-\int_{N_{\text {initial }}}^{N} \mathrm{~d} N=\frac{A_{0}}{\beta} \int_{T_{\text {initial }}}^{T} \exp \left(-\frac{E_{\text {des }}}{R T}\right) \mathrm{d} T$

And, for a first-order desorption process, the suitable expression is

$-\int_{N_{\text {initial }}}^{N} \frac{\mathrm{d} N}{N}=\frac{A_{1}}{\beta} \int_{T_{\text {initial }}}^{T} \exp \left(-\frac{E_{\mathrm{des}}}{R T}\right) \mathrm{d} T$
Table 6. Values for the parameters used for the simulation.

\begin{tabular}{|c|c|c|c|}
\hline Molecule & Parameter & Value & Units \\
\hline \multirow{6}{*}{ Methanol } & $E_{\operatorname{des}_{0}}$ & 46.2 & $\mathrm{~kJ} \mathrm{~mol}^{-1}$ \\
\hline & $A_{0}$ & $1.94 \times 10^{31}$ & molecule $\mathrm{cm}^{-2} \mathrm{~s}^{-1}$ \\
\hline & $E_{\mathrm{des}_{1}}$ & 46.4 & $\mathrm{~kJ} \mathrm{~mol}^{-1}$ \\
\hline & $A_{1}$ & $5.0 \times 10^{14}$ & $\mathrm{~s}^{-1}$ \\
\hline & $N($ initial) & $5.5 \times 10^{15}$ & molecule $\mathrm{cm}^{-2}$ \\
\hline & $\beta$ & 1 & $\mathrm{~K}_{\text {century }}{ }^{-1}$ \\
\hline \multirow{4}{*}{ Water } & $E_{\mathrm{des}_{0}}$ & 47.0 & $\mathrm{~kJ} \mathrm{~mol}^{-1}$ \\
\hline & $A_{0}$ & $9.1 \times 10^{29}$ & molecule $\mathrm{cm}^{-2} \mathrm{~s}^{-1}$ \\
\hline & $N$ (initial) & $1.15 \times 10^{17}$ & molecule $\mathrm{cm}^{-2}$ \\
\hline & $\beta$ & 1 & $\mathrm{~K}_{\text {century }}{ }^{-1}$ \\
\hline Wat.:Meth. & Proportion & 100:5 & \\
\hline Meth. $_{0}:$ Meth. 1 & Proportion & $40: 60$ & \\
\hline
\end{tabular}

Subindex ' 0 ' refers to a zeroth-order desorption process.

Subindex ' 1 ' refers to a first-order desorption process.

In Table 6, all the parameters necessary to perform the simulation for the molecules methanol and water are shown. The parameters with a subscript ' 0 ' are related to a zeroth-order desorption process, and those with a subscript ' 1 ' are referred to a first-order desorption process. To find the best value of $A_{0}$ to fit the experimental results, we have only considered the interval of temperatures used for our experiments (144-152 K). $N$ (initial) for water has been used to represent the assumed amount in these environments (Hasegawa \& Herbst 1993; Fraser et al. 2001). Na(initial) for methanol has been calculated from the proportion of water to methanol (100:5) used by Collings et al. (2004) in their experiments. The parameters $E_{\mathrm{des}_{1}}$ (desorption energy for a first-order process related to methanolwater binding), $A_{1}$ (frequency factor for a first-order process) and Meth $_{0}:$ Meth $_{1}$ (proportion of methanol desorbing as a zeroth-order versus methanol desorbing as a first-order process) were derived to reproduce the profile obtained by Collings et al. (2004) for layered desorption during their experiments.

Fig. 9 (top panel) shows the theoretical desorption profile of the mixture methanol and water (100:5) if methanol is present in the outer layer of the ices on the dust grains of hot cores. Two different desorption peaks can be observed; the first one, at $94 \mathrm{~K}$, corresponds to the external layers of methanol due to methanol-methanol interaction, and the second desorption peak, at $99 \mathrm{~K}$, corresponds to the internal layers of methanol where methanol-water binding energy is assumed. Finally, water desorbs at $108 \mathrm{~K}$. Regarding the evolution of the coverage of icy species (bottom panel), the external layers of methanol completely desorb at $94 \mathrm{~K}$, and the internal layers desorb to the gas phase at $102 \mathrm{~K}$.

Fig. 10 shows the evolution of the same mixture if methanol is co-deposited with water. In this case, only a desorption peak for methanol is observed (top panel), in agreement with the experimental results of Collings et al. (2004) and Martín-Doménech et al. (2014). This peak corresponds to the desorption of water and represents the presence of methanol in the proportion 100:5 (or 100:3 in the case of Martín-Doménech et al. 2014) because molecules of methanol are trapped within the water matrix. Under these conditions, the methanol desorbs between 100 and $108 \mathrm{~K}$, together with water.

It is worth noting that the resulting peaks of this simulated desorption appear at temperatures lower than those obtained experimentally by Collings et al. (2004) due to the different warming rates as can be expected from Fig. 11. 

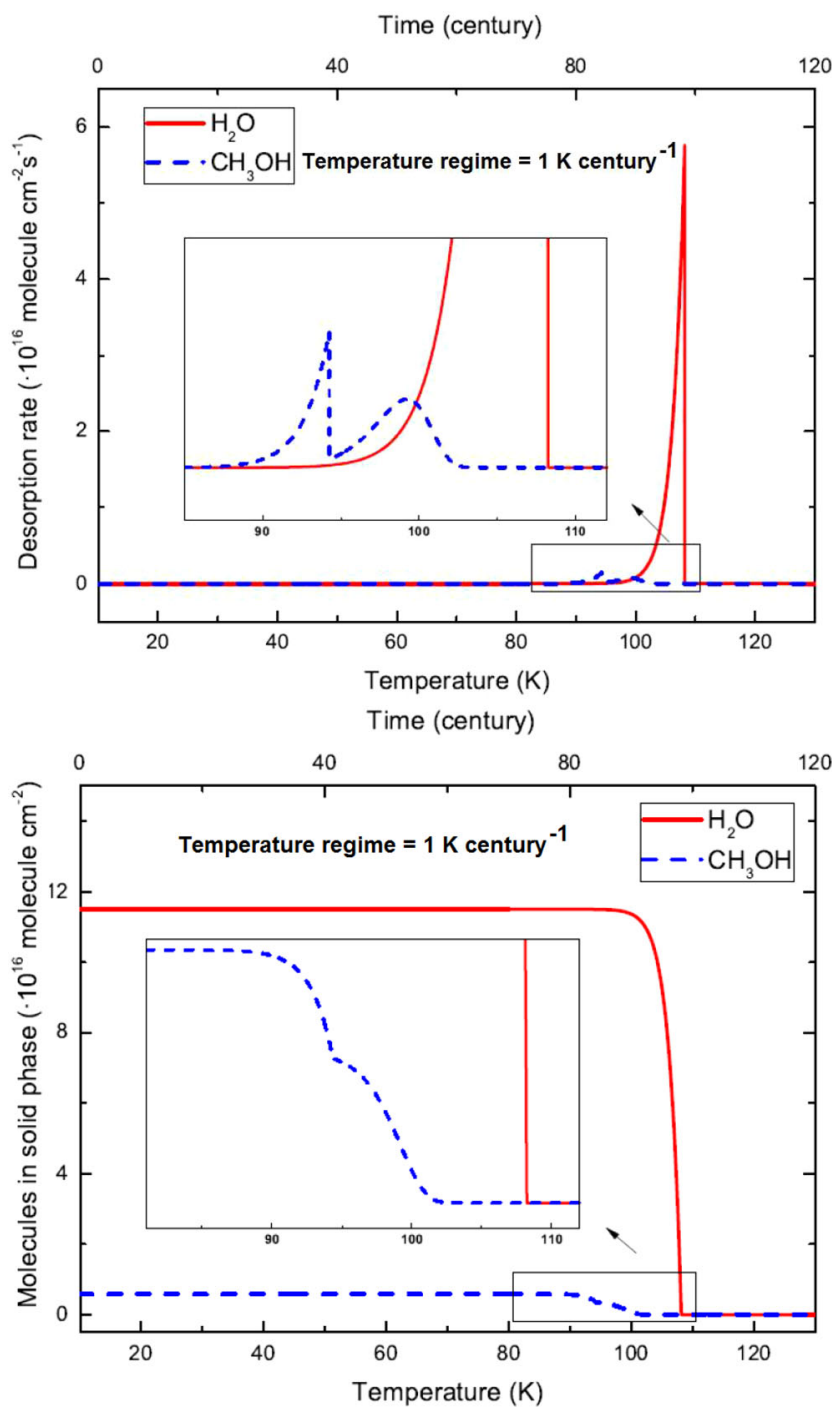

Figure 9. Simulation of the desorption process evolution for methanol and water if methanol is formed in the outer layer of water taking $\beta=1$ Kcentury $^{-1}$. Desorption rate (top panel) and molecules in solid phase (bottom panel).

\section{ASTROPHYSICAL IMPLICATIONS}

Hot cores present a large diversity of ice compositions and abundances since each source present different physical conditions of warming, UV irradiation, flow shocks, etc. Methanol is considered the precursor of many COMs in these scenarios (Garrod \& Widicus Weaver 2013; Öberg et al. 2013; Bertin et al. 2016). If these organic molecules are formed in solid phase induced by UV irradiation (Muñoz-Caro et al. 2002; Öberg et al. 2009), they are constrained by the availability of methanol in this phase. The temperature of the dust is a determining factor since this molecule desorbs depending on the temperature in these scenarios. Therefore, knowing the desorption temperature for methanol under these conditions is necessary to understand why different cores present different proportions of this molecule. Nevertheless, similar presence of COMs are detected in high-, intermediate and low-mass hot cores. Öberg et al. (2011b) have calculated the average abundance of methanol for different low- and high-mass sources, resulting in a similar value of 7 and 8 per cent relative to water.
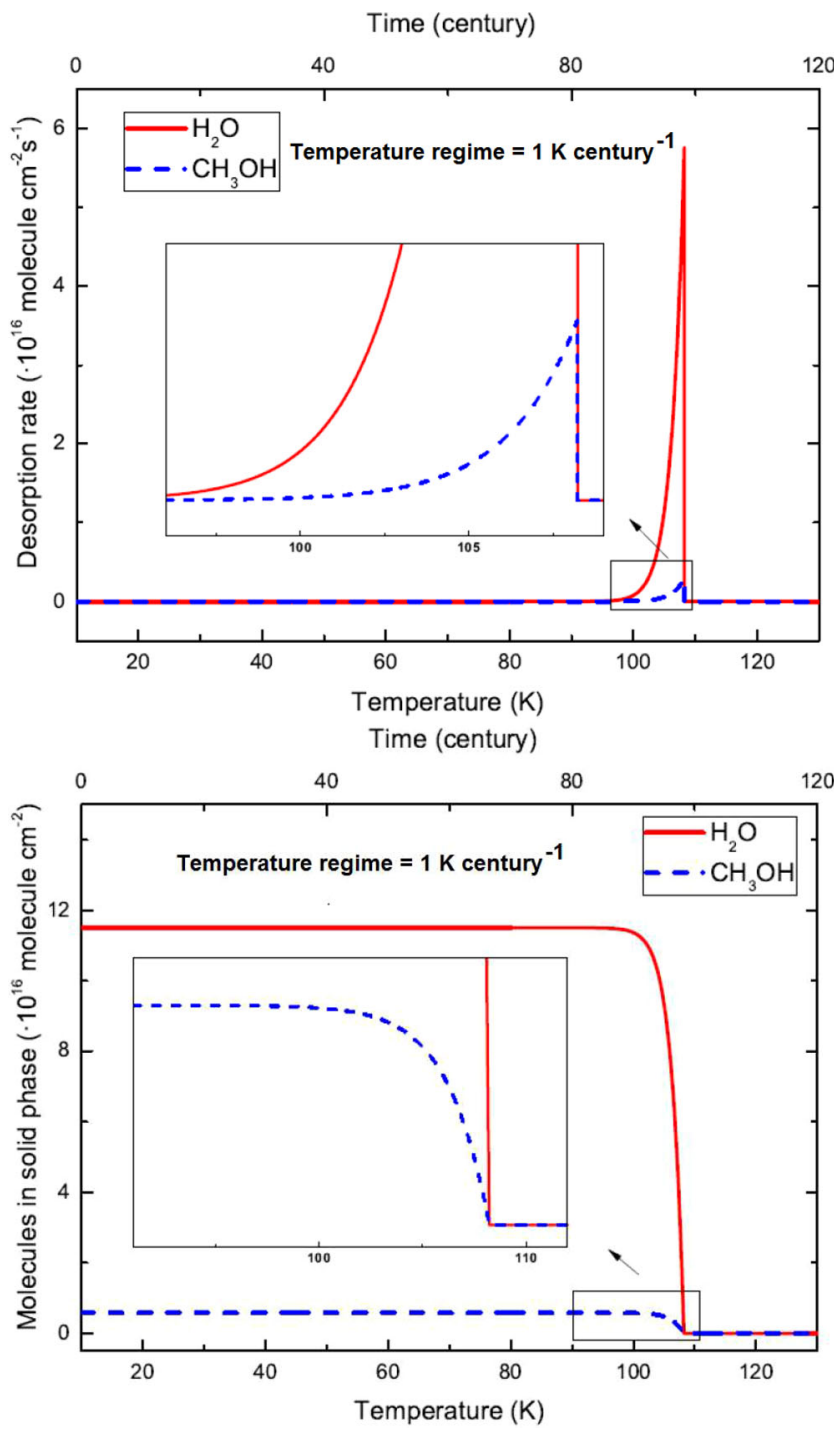

Figure 10. Simulation of the evolution desorption process of methanol and water if methanol and water are co-deposited taking $\beta=1$ Kcentury $^{-1}$. Desorption rate (top panel) and molecule in solid phase (bottom panel).

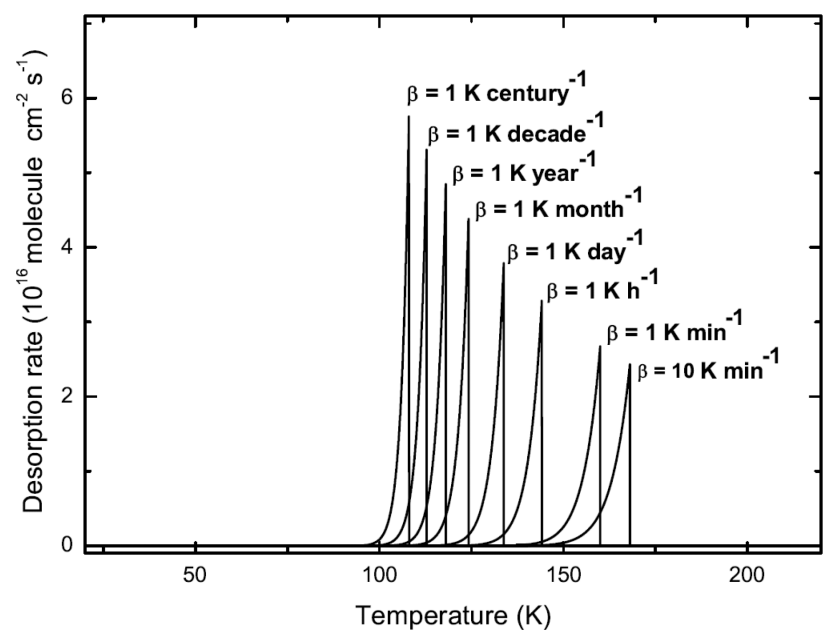

Figure 11. Simulation of a non-equilibrium desorption of 100 monolayers of water for several values of $\beta=\mathrm{d} T / \mathrm{d} t$. 
Methanol is frequently mixed with water ice in the icy mantles on dust grains. However, in some young stellar objects of the Serpens Molecular Cloud, the analysis of the methanol:water correlation suggests the presence of methanol ice segregated from water (Cuppen et al. 2011; Whittet et al. 2011). The results obtained in Section 3 concerning pure methanol ice can be applicable to these environments where pure methanol suffers a thermal process.

Table 5 shows values of half-life for methanol at different temperatures related to astrophysical environments. To interpret these results, a period of $10^{2} \mathrm{yr}$ is usually taken as a reference, and is considered to be an appreciable time for residence on the surface of dust grains in astrophysical conditions (Brown \& Bolina 2007). Therefore, for pure methanol, the temperature necessary to desorb at least a 50 percent of its initial amount of molecules in $10^{2} \mathrm{yr}$ varies from 80 to $110 \mathrm{~K}$ from different authors, as can be inferred from Table 5.

Taking into account that grain lifetimes are calculated to be in the range 0.4-2.2 Gyr (Jones et al. 1994), it can be inferred from the desorption obtained for all the authors present in Table 5 that for scenarios with temperatures lower than $60 \mathrm{~K}$ it would not be possible to detect gas phase methanol if its presence is related to a thermal process. Sandford \& Allamandola (1993) claimed that all the molecules should be depleted from the gas phase on to the cold grain surface in the dense ISM grains, in the absence of effects other than the thermal one. Then, to explain how it can be possible to observe those molecules in the gas phase in many lines of sight, some other energy inputs have to be considered, such as for example stellar jets impact on distant molecular clouds (Girart et al. 2005). Additional interaction producing desorption of icy components are related to $\mathrm{H}, \mathrm{Ni}$ and $\mathrm{Fe}$ ions. Seperuelo-Duarte et al. (2010) state that the effect of heavy ions is at least as important as the effect of protons in the interstellar medium. UV photodesorption seems to be negligible from the study of Bertin et al. (2016). Another source of energy which can be considered to explain the presence of the gas phase of these molecules in cold environments could be grain collisions (d'Hendecourt, Allamandola \& Greenberg 1982) or cosmic rays (Leger, Jura \& Omont 1985).

Concerning the mixture methanol:water, laboratory desorption experiments of this mixture (layered or co-deposited) lead to a desorption temperature ranging in the interval 160-180 K (Collings et al. 2004; Martín-Doménech et al. 2014). However, if the kinetics parameters of both molecules are calculated and then co-desorption is simulated at a warming rate of $1 \mathrm{~K}$ century ${ }^{-1}$ the desorption temperature lies in the interval $95-108 \mathrm{~K}$.

The methanol column density for several young massive stars were calculated by van der Tak et al. (2000). They found a jump in its concentration in the gas phase at $T \sim 100 \mathrm{~K}$, which suggests that this enhancement is produced by evaporation of icy grain mantles. In the case of the objects W 33A, GL 2591 and NGC 7358 IRS1, they have measured the concentration of methanol for cold $(T<90 \mathrm{~K})$ and warm $(T>90 \mathrm{~K})$ gas. The values for the concentration of methanol increase by a factor of $\sim 30$ from the cold gas to the warm one. This transition temperature coincides with the temperature theoretically obtained in this work for the desorption of the layered methanol:water ice.

Taking into account both possible thermal desorption models simulated (layered and co-deposited), it would be necessary to achieve a temperature of at least $94 \mathrm{~K}$ to explain the presence of methanol in the gas phase because this molecule does not desorb at a lower temperature in any of the models implemented.

Methanol would only be present in the gas phase at $94 \mathrm{~K}$ if it was in the outer layers of the ice surrounding the dust grains. If methanol is not observed in the gas phase even though the temperature of the hot core is evaluated to be between 94 and $99 \mathrm{~K}$, the presence of this molecule in the solid phase is not necessarily discarded because it could be trapped in the water matrix; this fact would imply a previous input of energy, as mentioned above, and further co-deposition.

Finally, if methanol is observed in the gas phase at temperatures lower than $94 \mathrm{~K}$, it necessarily implies some different energy input to explain its presence as has been pointed out in the case of pure methanol.

\section{SUMMARY AND CONCLUSIONS}

Desorption energy and frequency factor are two parameters widely used in astrophysics when desorption of ices is studied. Obtaining these kinetics parameters for methanol helps to better understand its solid-gas phase composition in many astrophysical scenarios.

In this paper, new results for the desorption energy and frequency factor for methanol and water are presented. For the first time, the variation of the frequency factor with temperature has been addressed in order to know whether this influence is significant and to obtain a more representative value for this parameter.

The method implemented to determine desorption energy and the frequency factor is different from those previously published. The experiments performed in this work are based on the variation of the frequency signal of a QCMB when an ice is desorbing from its surface after multilayer deposition. In this work, zeroth-order kinetics are assumed, supported by comparing the experimental desorption profile and the theoretical one. It is important to emphasize that using the experimental set-up presented here, the molecules released from the substrate (also acting as a microbalance) are directly measured, making it possible to perform this type of studies under HV conditions.

The results obtained for the kinetics parameters for pure methanol have been compared to those previously reported in the literature. Additionally, they have been used to obtain residence time at several temperatures of astrophysical interest to compare the results obtained in this work with those calculated with the parameters reported by other authors. The similarities obtained validate the method presented here. Nevertheless, when the effect of temperature on the pre-exponential factor is taken into account, more accurate results on desorption simulation are obtained.

Additionally, the mixture of methanol and water ice present on dust grains in hot cores of massive stars has been addressed. The study of the desorption of this mixture is necessary to interpret observational results since methanol frequently interacts with water in astrophysical scenarios.

Once the kinetics of the desorption process of each ice has been determined, it has been simulated in the desorption process of the mixture of water and methanol (100:5) taking into account the results obtained by Collings et al. (2004) and Martín-Doménech et al. (2014) using layered or co-deposited mixture of methanol and water.

The theoretical desorption profile obtained in this work simulates the warming conditions present in the hot cores of massive young stars $\left(1 \mathrm{~K}\right.$ century $\left.{ }^{-1}\right)$. As this warming rate is much lower than the values used in the laboratory, the desorption peak appears at temperatures around 95-108 K. These desorption temperatures are lower than the experimental results of Collings et al. (2004), and Martín-Doménech et al. (2014) (160-180 K), since this temperature depends on the warming rate. 
The observational results of some massive young objects (van der Tak et al. 2000) lead to a desorption temperature around 90$100 \mathrm{~K}$ which also agrees with the value obtained with the theoretical model presented here for layered methanol:water ices and confirms its validity.

From these results, some implications can be drawn from the detection or non-detection of methanol in the gas phase when hot cores are observed. If methanol is detected in the gas phase at temperatures lower than $94 \mathrm{~K}$, a different energy input can be inferred.

\section{ACKNOWLEDGEMENTS}

This work was supported by the Ministerio de Economía y Competitividad FIS 2013-48087-C2-2-P. We want to thank the Departamento de Ingeniería Textil y Papelera de la Escuela Politécnica Superior de Alcoy for supplying us with pure water.

\section{REFERENCES}

Bertin M. et al., 2016, ApJ, 817, L12

Bolina A. S., Wolff A. J., Brown W. A., 2005, J. Chem. Phys., 122, 044713 Bottinelli S. et al., 2004, ApJ, 615, 354

Brown W. A., Bolina A. S., 2007, MNRAS, 374, 1006

Charnley S. B., Tielens A. G. G. M., Rodgers S. D., 1997, ApJ, 482, L203

Collings M. P., Anderson M. A., Chen R., Dever J. W., Viti S., Williams D. A., McCoustra M. R. S., 2004, MNRAS, 354, 1133

Cruz-Diaz G. A., Martín-Doménech R., Muñoz Caro G. M., Chen Y.-J., 2016, A\&A, 592, A68

Cuppen H. M., Penteado E. M., Isokoski K., van der Marel N., Linnartz H., 2011, MNRAS, 417, 2809

d'Hendecourt L. B., Allamandola L. J., Greenberg J. M., 1982, A\&A, 109, L12

Dartois E., Demyk K., d'Hendecourt L., Ehrenfreund P., 1999, A\&A, 351, 1066

Doronin M., Bertin M., Michaut X., Philippe L., Filion J. H., 2015, J. Chem. Phys., 143, 084703

Fraser H. J., Collings M. P., McCoustra M. R. S., Williams D. A., 2001, MNRAS, 327, 1165

Fuchs G. W., Cuppen H. M., Ioppolo S., Romanzin C., Bisschop S. E., Andersson S., van Dishoeck E. F., Linnartz H., 2009, A\&A, 505, 629

Galwey A. K., Brown M. E., 2002, Thermochim. Acta, 386, 91

Garrod R. T., Widicus Weaver S. L., 2013, Chem. Rev., 113, 8939

Gibb E. L, Whittet D. C. B., Broogert A. C. A., Tielens A. G. G. M., 2004, ApJS, 151, 35

Girart J. M., Viti S., Estalella R., Williams D. A., 2005, A\&A, 439, 601
Hasegawa T. L., Herbst E., 1993, MNRAS, 261, 83

Jones A. P., Tielens A. G. G. M., Hollenbach D. J., McKee C. F., 1994, ApJ, 433, 797

Kama M. et al., 2010, A\&A, 521, L39

Leger A., Jura M., Omont A., 1985, A\&A, 144, 147

Luna R., Millán C., Santonja C., Satorre M. Á., 2009, Vacuum, 83, 942

Luna R., Millán C., Domingo M., Satorre M. Á., Santonja C., 2015, Vacuum, 122,154

Martín-Doménech R., Muñoz Caro G. M., Bueno J., Goesmann F., 2014, A\&A, 564, A8

Martín-Doménech R., Muñoz-Caro G., Cruz-Diaz G. A., 2016, A\&A, 589, A107

Muñoz-Caro G. M. et al., 2002, Nature, 416, 403

Öberg K. I., Garrod R. T., van Dishoeck E. F., Linnartz H., 2009, A\&A, 504 , 891

Öberg K. I., van der Marel N., Kristensen L. E., van Dishoeck E. F., 2011a, ApJ, 740, 14

Öberg K. I., Boogert A. C., Pontoppidan K. M., van den Broek S., van Dishoeck E. F., Bottinelli S., Blake G. A., Evans N. J., II, 2011b, ApJ, 740, 109

Öberg K. I., Boamah M. D., Fayolle E. C., Garrod R. T., Cyganowski C. J., van der Tak F., 2013, ApJ, 771, 95

Polanyi M., Wigner E., 1925, EZPhysik, 33, 429

Pontoppidan K. M., Dartois E., van Dishoeck E. F., Thi W. F., d'Hendecourt L., 2003, A\&A, 404, L17

Pontoppidan K. M., van Dishoeck E. F., Dartois E., 2004, A\&A, 426, 925

Requena-Torres M. A., Martín-Pintado J., Martín S., Morris M. R., 2008, ApJ, 672, 352

Sandford S. A., Allamandola L. J., 1993, ApJ, 417, 815

Seperuelo-Duarte E., Domaracka A., Boduch P., Rothard H., Dartois E., da Silveira E. F., 2010, A\&A, 512, 71

Smith R. S., Matthiensen J., Kay B. D., 2014, Phys. Chem., 118, 8242

Tielens A. G. G. M., Whittet D. C. B., 1997, in van Dishoeck E. F., ed., Molecules in Astrophysics: Probe and Process. Kluver, Dordrecht

van der Tak F. F. S., van Dishoeck E. F., Caselli P., 2000, A\&A, 361, 327

Viti S., Collings M. P., Dever J. W., McCoustra M. R. S., Williams D. A., 2004, MNRAS, 354, 1141

Watanabe N., Kouchi A., 2002, ApJ, 571, L173

Whittet D. C. B., Chiar J. E., 1993, A\&AR, 5, 1

Whittet D. C. B., Cook A. M., Herbst E., Chiar J. E., Shenoy S. S., 2011, ApJ, 742, 28

This paper has been typeset from a $\mathrm{T}_{\mathrm{E}} \mathrm{X} / \mathrm{LT}_{\mathrm{E}} \mathrm{X}$ file prepared by the author. 